\title{
Urban network travel time estimation from stop-line loop detector data and signal controller data
}

\author{
A. Bhaskar ${ }^{1}$, E. Chung $^{1}$, M. Kuwahara ${ }^{2}$, O. de Mouzon ${ }^{3}$ \\ \& A.-G. Dumont ${ }^{1}$ \\ ${ }^{1}$ Traffic Facilities Laboratory (LAVOC), \\ Swiss Federal Institute of Technology, Lausanne, Switzerland \\ ${ }^{2}$ Institute of Industrial Science, The University of Tokyo, Tokyo, Japan \\ ${ }^{3}$ Traffic Engineering Laboratory (LICIT), \\ French National Institute for Transport and Safety Research (INRETS), \\ Lyon-Bron, France
}

\begin{abstract}
This paper presents a model to estimate travel time using cumulative plots. Three different cases are considered: i) case-Det, for only detector data; ii) case-DetSig, for detector data and signal controller data and iii) case-DetSigSFR, for detector data, signal controller data and saturation flow rate. The performance of the model for different detection intervals is evaluated. It is observed that detection interval is not critical if signal timings are available. Comparable accuracy can be obtained from larger detection interval with signal timings or from shorter detection interval without signal timings. The performance for case-DetSig and for case-DetSigSFR is consistent with accuracy generally more than $95 \%$ whereas case-Det is highly sensitive to the signal phases in the detection interval and its performance is uncertain if detection interval is an integral multiple of signal cycles.

Keywords: urban network travel time, arterial travel time, cumulative plots, signalised intersection, delay.
\end{abstract}

\section{Introduction}

Travel time information is one of the important performance measures for the transportation systems. In the literature, researchers had proposed different 
methodologies for travel time estimation ranging from simple regression to more sophisticated artificial intelligence based algorithms. Most of the research on travel time estimation is limited to freeways [1, 2]. In urban environment, the task is more challenging. The presence of conflicting areas whether signalised or non-signalised imposes delay that has a significant affect on travel time estimation. Generally, in most of the signalised intersections the primary use of detectors is for signal control. There has been increasing interest to utilise detector data for travel time estimation such as developing the regression relationship between detector occupancy and mean intersection delay with use of traffic simulation modelling [3-5] or real data [6]. Sisiopiku and Rouphail [7] provide a review of earlier work on the use of detector output for travel time estimation. The regression analysis models provide a statistical relationship between the variables without explicitly addressing the traffic processes that determine these travel times. These models are often site specific and require calibration of parameters for real application. Moreover, the regression analysis is observed for detectors far from the stop-line and cannot be applied for stopline detectors.

The model presented in this paper integrates stop-line loop detector data with signal controller data and considers saturation flow rate. The model is expected to be a valuable tool for system performance evaluation and for estimating level of service for different intersections. It can also be applied for ITS applications such as Advanced Traveller Information Systems and dynamic route guidance.

\section{Travel time estimation model}

The model considers classical analytical procedure of using cumulative plots for travel time estimation. In Figure 1, a single lane link (study link) between two consecutive intersections is shown. Travel time is defined as the time difference between the time when the vehicle enters the downstream intersection and the time when it entered the upstream intersection. The area $(A)$, between cumulative plot at the upstream end of the link $\left(C P_{U S}(t)\right)$ and cumulative plot at the downstream end of the link $\left(C P_{D S}(t)\right)$ provides the total experienced travel time for all the $N$ number of vehicles which arrived during $\Delta t$ time interval at the upstream end of the link. Average travel time per vehicle is the ratio between total area and number of vehicles arrived.

In this research, it is assumed that there is no shared lane and the stop-line detectors are present on all the lanes that contribute to cumulative plots. Stopline loop detectors, provide aggregated counts and occupancy during detector detection interval. Signal controller provides real time signal parameters such as signal phase plan and start time and end time of each phase. Saturation flow rate is a parameter specific to each site under study. To generalise the model, cumulative plots at the location of the detector are estimated for the three cases depending on the availabilities of the data a) Case-Det: Only detector data is available; b) Case-DetSig: Detector data and signal controller data is available and c) Case-DetSigSFR: Detector data, signal controller data and saturation flow rate is available. The slope of the plot defines the flow pattern at the respective entrance of the intersection. 


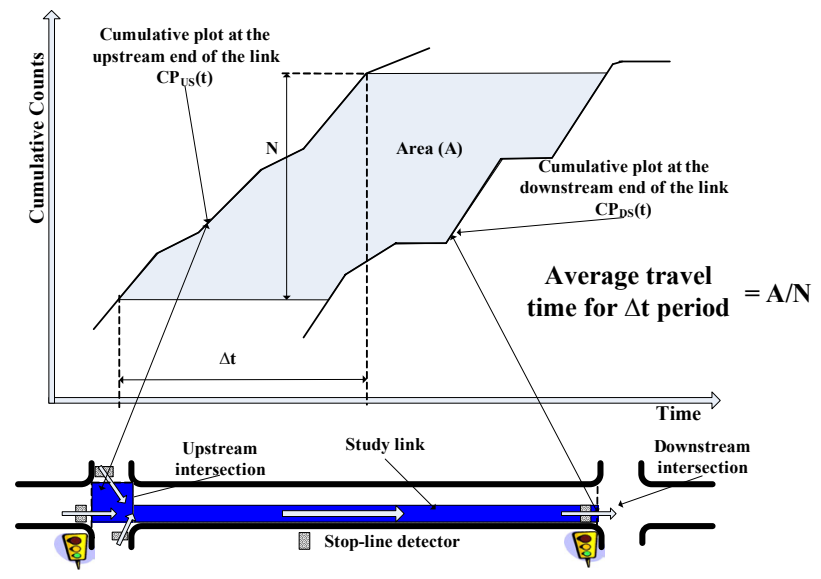

Figure 1: Analytical methodology for travel time estimation.

We define $N^{d}$ and $\mathrm{q}$ as the counts and flow, respectively during the detection interval of $D I$ seconds.

Case-Det: If only detector data is available then it is assumed that the counts during a detection interval are from a flow pattern (eqn (1)) that is uniformly distributed throughout the detection interval (Figure 2(a)). The assumption is reasonable for lower detection intervals and in the absence of any further information can be applied for higher detection interval.

$$
q=\frac{N^{d}}{D I}
$$
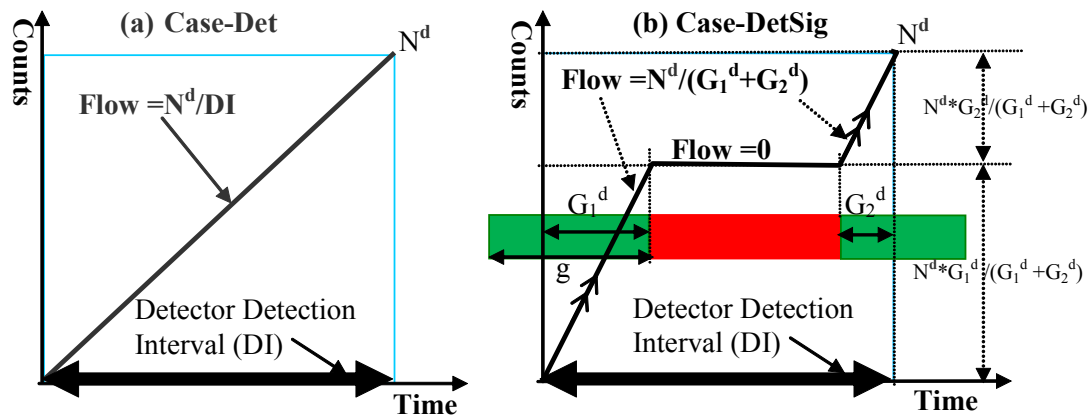

Figure 2: $\quad$ Flow pattern for (a) case- Det and (b) case-DetSig.

Case-DetSig: If detector data and signal controller data is available then it is assumed that the counts during a detection interval are from a stepwise flow pattern (eqn (2)). Flow pattern is uniform only during the green period of the detection interval and during red period there is no flow (Figure 2(b)). This captures the fluctuations in the flow pattern even for higher detection interval. We define $G_{i}^{d}$ as the $i$-th green period during the detection interval In 
Figure 2(b), two green periods are present during the detection interval and the counts are distributed to each green interval in proportion to the corresponding green time. Flow pattern during each green periods of the detection interval are parallel to each other.

$$
\begin{aligned}
q & =\frac{N^{d}}{\sum_{G_{i}}{ }^{d}} \text { during green period in detection interval } \\
& =0 \quad \text { during red period in detection interval }
\end{aligned}
$$

Case-DetSigSFR: We define the demand, which is the cumulative plot $\left(C P_{\text {demand }}\right)$ at the location of the stop-line detector if there was no restriction on the flow of the vehicles. At a signalised intersection, during the green phase, the vehicles from the queue are effectively discharged at saturation flow. Thereafter, the flow pattern follows the demand pattern. If saturation flow pattern is known then more accurate flow pattern considering saturation flow and non-saturation flow can be estimated.

The count, $N_{i}$, during each $i$-th green interval in the detection interval is assumed to be in proportion to the corresponding green period (eqn (3)).

$$
N_{i}=\frac{N^{d} * G_{i}^{d}}{\sum_{i} G_{i}^{d}}
$$

For simplicity, we focus on effective green $(g)$ for a complete signal cycle instead of green periods $\left(G_{i}^{d}\right)$ in each detection interval. The effective green can extend in more than one detection interval. For instance, in Figure 2(b), the effective green $g$ has the component $G_{l}{ }^{d}$ during the detection interval indicated in the figure. The count, $N_{g}$, for effective green is obtained by respective adding the counts from all its components, if split in more than one detection intervals. The maximum number of vehicles which can depart during the effective green time is $N_{\max }\left(={ }^{*} *_{g}\right)$, where $s$ is saturation flow rate (vehicles/second). Out of $N_{g}$ vehicle, $n_{s}$ number of vehicles enters the intersection at saturation flow pattern and the remaining $\left(N_{g}-n_{s}\right)$ follow the demand pattern.

For a link between two consecutive intersections as shown in Figure 1, the demand pattern for the detector at the downstream end of the link can be estimated from $C P_{U S}(t)$. However, for a network there can be certain links where the $C P_{U S}(t)$ is unknown such as at the entrance of the network. Therefore, the following two cases of unknown or know demand pattern are considered to estimate cumulative plots for case-DetSigSFR.

(a) Unknown demand pattern: The detector counts represent demand for undersaturated and saturated situations. However, for over-saturated situation, the counts are upper bounded by capacity and that may be less than true demand. Therefore, demand estimated in this case is termed as "assumed demand".

The demand flow pattern can be assumed to follow a uniform pattern (deterministic) or can be assumed to be distributed according to some probability distribution (stochastic). To simplify the analysis it is assumed that demand is uniform during the signal cycle. As shown in Figure 3, $N_{g}$ numbers of vehicles 
are counted during the green period that represents the uniform demand for the signal cycle. By superimposing saturation flow pattern during the green period on the uniform demand pattern the following relationship can be geometrically obtained:

$$
\begin{array}{rlrl}
\frac{n_{s}}{N_{g}}= & \frac{(1-g / C)}{\left(1-\frac{N_{g}}{N_{\max }} \frac{g}{C}\right)} & & \text { for } N_{g} \leq N_{\max } \\
& =1 & \text { for } N_{g}>N_{\max }
\end{array}
$$

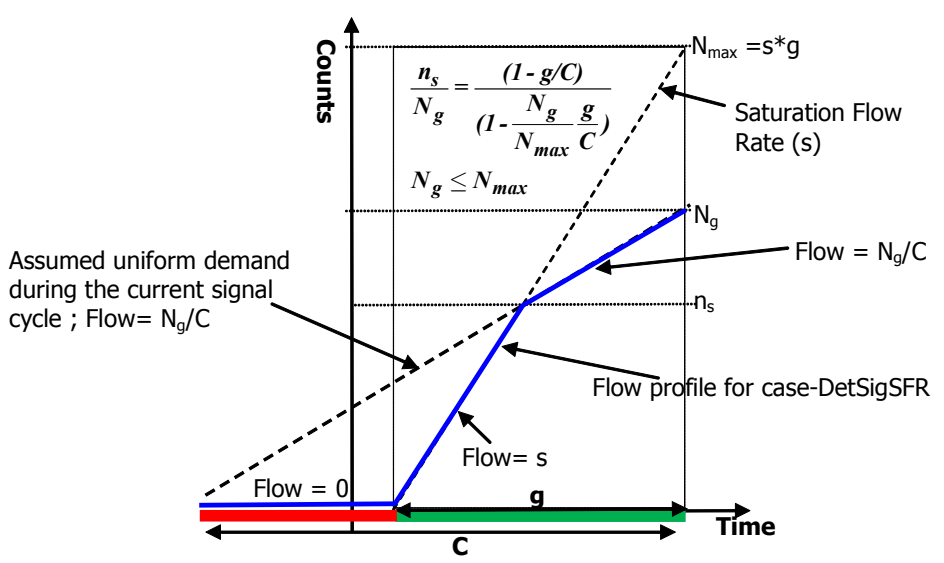

Figure 3: Geometrical relationship between $n_{s}$ and $N_{g}$ assuming uniform demand pattern during the current signal cycle.

(b) Known demand pattern: In this case, we are interested to estimate $C P_{D S}(t)$, given $C P_{U S}(t)$. The $C P_{\text {demand }}(t)$ is the horizontal shift of the $C P_{U S}$ by free-flow travel time $\left(t t_{\text {freeflow }}\right)$ of the link i.e., $C P_{U S}\left(t-t t_{\text {freeflow }}\right)$. The demand is deduced from the upstream cumulative plot, so we call this demand as "deduced demand". It is assumed that the vehicle counts during the detection interval are from a stepwise flow pattern. The flow is zero for red intervals and for green interval if $C P_{\text {demand }}$ is greater than the cumulative counts $\left(C P_{D S}(t)\right)$ then the flow is at saturation flow otherwise, the flow pattern is same as demand pattern (see eqn (5)).

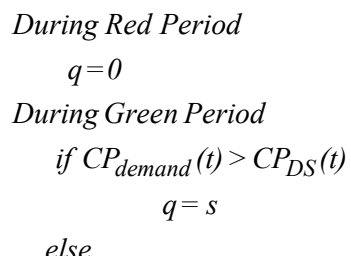

$$
q=\frac{\partial C P_{\text {demand }}(t)}{\partial t}
$$

As shown in Figure 4, the upstream cumulative plot, $C P_{U S}(t)$, and the number of vehicles departing from the downstream end of the link $(N)$ are known and flow pattern at downstream intersection for the current detection interval is unknown. 
The required flow pattern during the current detection interval is obtained by no flow during red period ( $a$ to $b$ ) and during green period, the flow is at saturation flow pattern until demand pattern intersects the saturation flow pattern ( $b$ to $c$ ) and thereafter flow follows the demand pattern $(c$ to $d)$.

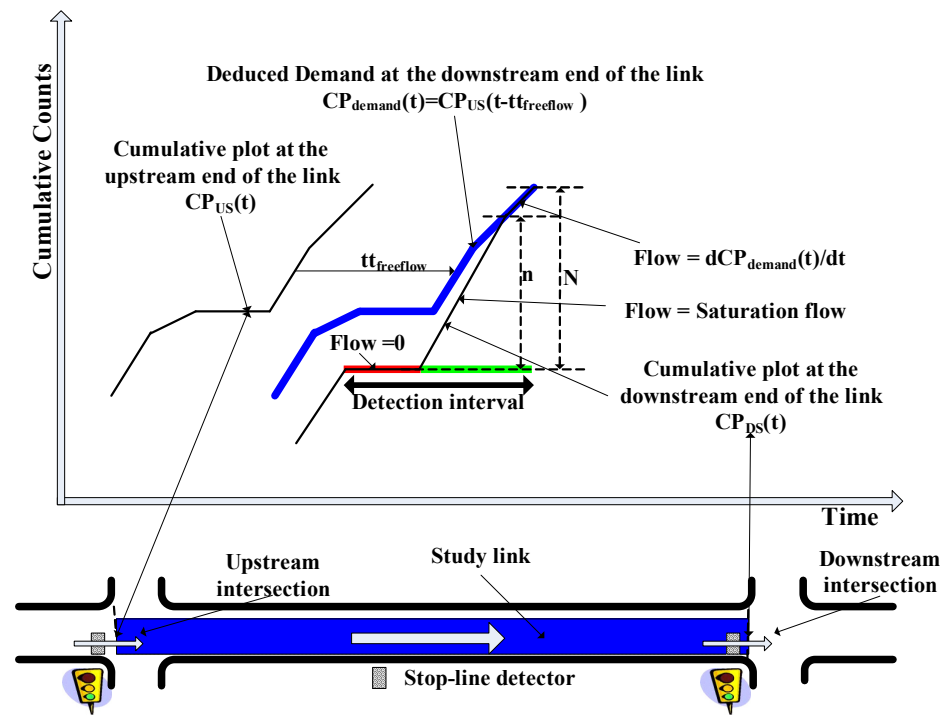

Figure 4: $\quad$ Flow pattern at downstream end of the link for case-DetSigSFR.

\section{Model testing on controlled environment}

The model is tested using a microscopic traffic simulator, AIMSUN where average estimated travel time is compared with the actual average travel time obtained from the individual vehicle. For simplicity, a test bed with only through movement at downstream intersection is developed. For upstream intersection the flow from three different directions are considered (as the link shown in Figure 1). Both the upstream and downstream intersections are signalised with bottleneck at the downstream intersection. Scenarios for different degrees of saturation in the range of 0.5 to 1.2 are simulated and average travel time for 6 minutes is considered. To avoid queue spill back, the length of the study link is around 1.1 kilometres. The sensitivity analysis for the model is performed for a fixed signal $($ cycle $=120$ seconds, green split $=0.5$ ) and detector detection interval of $15,30,60,90,120, \ldots, 330$ and 360 seconds. The performance of the model is defined in terms of accuracy (\%) as follows:

$$
\operatorname{Accuracy}(\%)=\left(1-\frac{\sum_{i=1}^{N} \frac{\mid \text { actual }_{i}-\text { estimated }_{i} \mid}{\text { actual }_{i}}}{N}\right) * 100
$$

where, $N$ is the total number of periods. Actual $_{i}$ and estimated $i$ are the average actual travel time and average estimated travel time for each period, respectively.

Figure 5 represents the graphs for detector detection intervals versus accuracy for the three cases. As expected, lower detection intervals have higher accuracy 
levels irrespective of the cases and for detection interval less than 30 seconds the estimation is very accurate (99.5\%). Detection interval is not critical if signal timings are available. Comparable accuracy can be obtained from a) detector data from larger detection interval with signal timings and $b$ ) detector data from shorter detection interval without signal timings. If detection interval is short, then signal controller data and saturation flow rate is not necessary. For caseDet, the performance is not consistent for different detection intervals and the accuracy is less than $80 \%$ when detection interval is an integral multiple of signal cycle for instance 120, 240 and 360 in this example. This inconsistency in the performance for case-Det is analysed in the next subsection. As expected, case-DetSigSFR performs better than case-DetSig because it more realistically represents the cumulative plots.

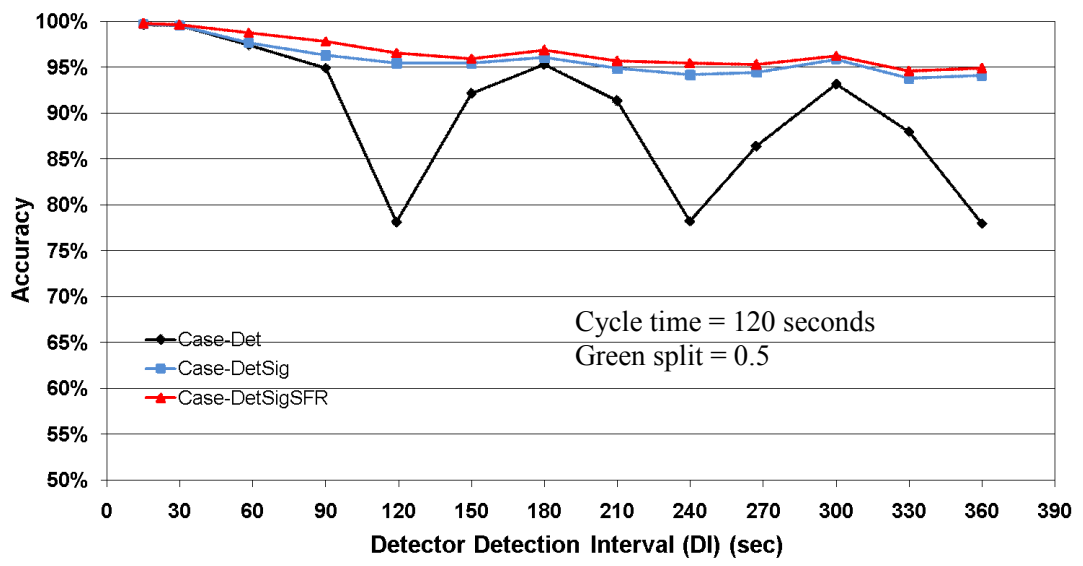

Figure 5: Detector detection interval versus accuracy.

\section{Fundamental analysis for case-Det and discussions}

Fluctuations in the flow from certain combinations of signal phases in the detection interval can result in significant error in the travel time estimation from the cumulative plots generated under case-Det. To study this, a detection interval equal to signal cycle is considered. Four different combinations of signal phases in the detection interval are possible: a) RG combination: red period followed by green period; b) GR combination: green period followed by red period; c) RGR combination: green period between two red periods; and d) GRG combination: red period between two green periods. As presented in Figure 6, for case-Det, $C P_{U S}(t)$ estimated for (a) RG combination has tendency to overestimate $(+)$ travel time and (b) GR combination has tendency to underestimate (-) travel time. If one draws similar figures for $C P_{D S}(t)$ estimated then (a) $\mathrm{RG}$ combination has tendency to underestimate (-) travel time and (b) GR combination has tendency to overestimate ( + ) travel time. For RGR and GRG combinations, the estimation for both $C P_{U S}(t)$ and $C P_{D S}(t)$ can be either underestimated, overestimated or exact. 
Figure 7 represents graph of actual average travel time versus estimated average travel time obtained from simulating following four different combinations of signal phases in the detection interval: a) RG_GR (++) combination b) GR_RG (--) combination c) GR_GR (-+) combination and d) RG_RG (+-) combination. Here, RG_GR (++) combination represents red phase followed by green phase in the detection interval at upstream intersection whereas, green phase followed by red phase in the detection interval at downstream intersection and so for others.

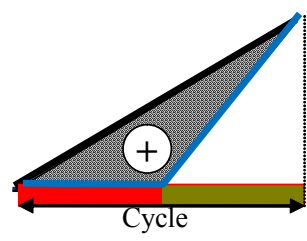

RG combination at the intersection

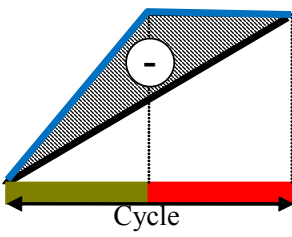

GR combination at the intersection

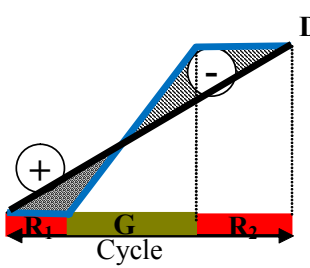

RGR combination at theintersection

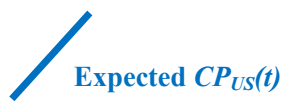

( Expected Overestimation

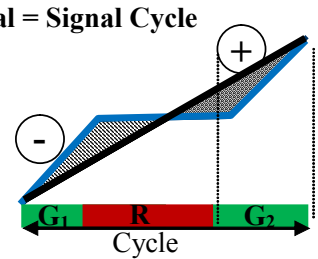

GRG combination at the intersection

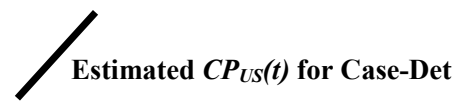

- Expected Underestimation

Figure 6: Estimated and expected flow pattern at upstream end of the link $\left(\mathrm{CP}_{\mathrm{US}}(\mathrm{t})\right)$ for case-Det.

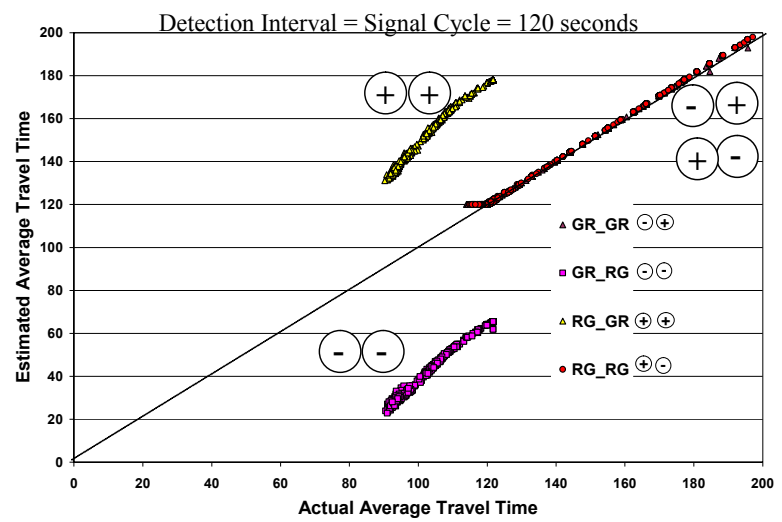

- Expected Underestimation

( Expected Overestimation

Figure 7: Performance for case-Det under different combination of signal phases in the detection interval. 


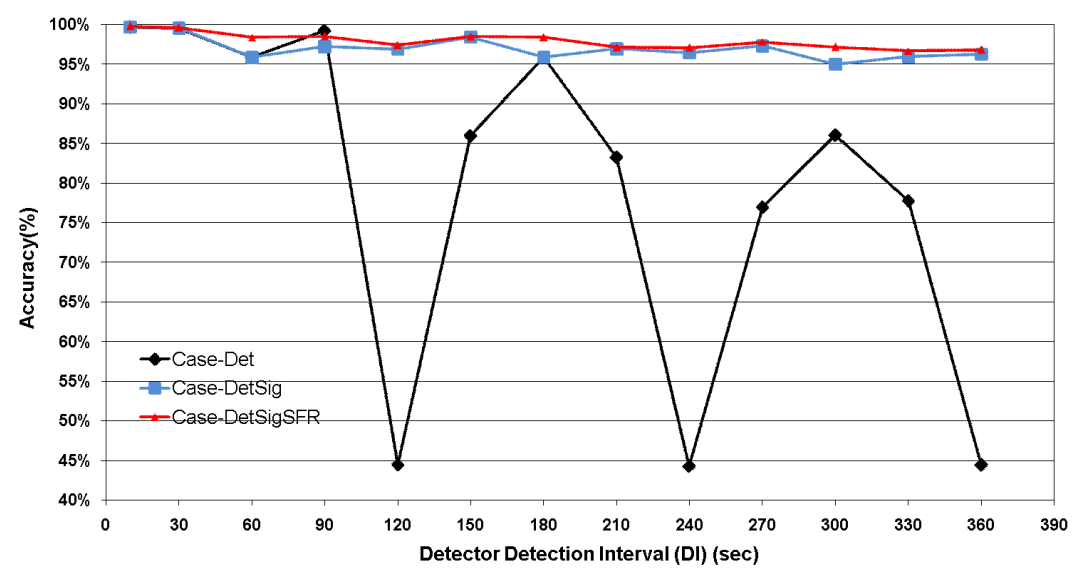

Figure 8: Detector detection intervals versus accuracy graphs for the three different cases of data availability with GR_RG (--) combination.

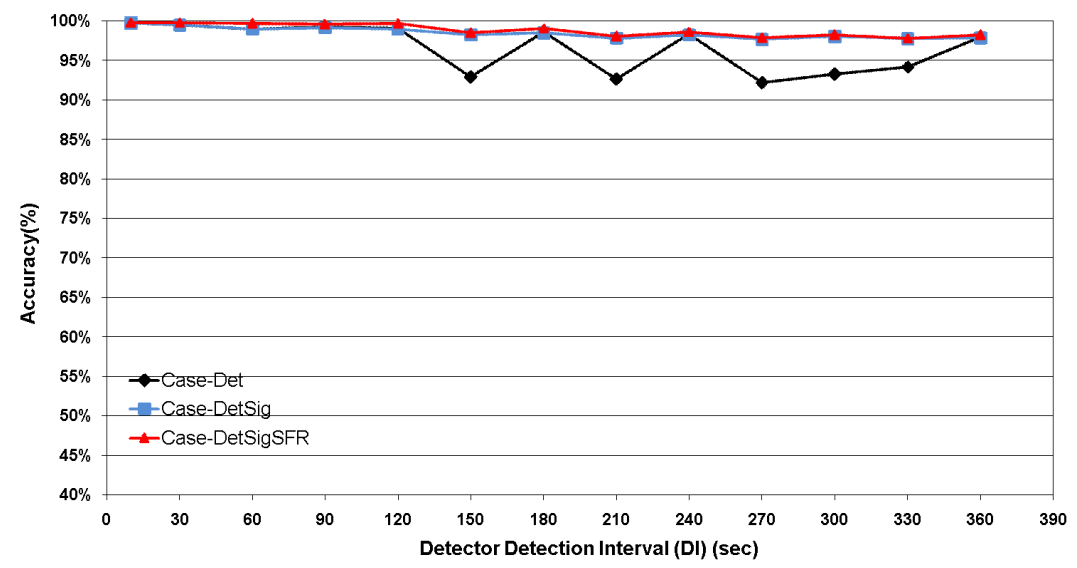

Figure 9: Detector detection intervals versus accuracy graphs for the three different cases of data availability with GR_GR (-+) combination.

The RG_GR $(++)$ combination has the tendency to highly overestimate travel time. Conversely, GR_RG (--) combination has the tendency to highly underestimate travel time. In the present example, the GR_GR (-+) and RG_RG (+-) combinations results in exact estimation because the underestimate travel time at upstream is compensated by the overestimate travel time at downstream and vice versa.

Figure 8 and Figure 9 present the detector detection intervals versus accuracy graphs for the three different cases for GR_RG(--) combination and GR_GR (-+) combination, respectively. The performance for case-Det is highly sensitive to the signal phases in the detection interval. When detection interval is integral multiple of signal cycles (120 sec, $240 \mathrm{sec}$ and $360 \mathrm{sec})$ then there is huge inconsistency in travel time estimation for case-Det. This means that when only 
detector data is available, detection interval should be carefully chosen in order to provide reliable travel time information from this data. The performance for case-DetSig and case-DetSigSFR is consistent and is not sensitive to the signal phases in the detection interval. The accuracy is generally more than $95 \%$ and is within the acceptable limits. The integration of detector data with signal controller has the potential to improve the accuracy with better confidence in estimation.

\section{Conclusions}

The model provides a reliable and good estimate for average travel time on a link between two consecutive signalised intersections. The performance of the model for different detection intervals indicated that for case-Det if the detection interval is integral multiple of signal cycle then the estimation can vary from perfect to worst. For case-Det, what matters is not how frequent the data is collected, but how the detection interval is related to signal timings. For instance, if signal cycle is two minutes and data is collected for four minutes interval then one can argue that for better confidence in travel time estimation one can collect the data for five minutes instead of four minutes which is twice the signal cycle.

\section{References}

[1] X. Zhang and J. A. Rice, "Short-term travel time prediction," Transportation Research Part C: Emerging Technologies, vol. 11, pp. 187-210, 2003.

[2] S. U. I. Bajwa, "Short-term travel time prediction using traffic detector data," in Department of Civil Engineering, Faculty of Engineers Tokyo, Japan: University of Tokyo, 2002, p. 99.

[3] V. P. Sisiopiku, N. M. Rouphail, and A. Santiago, "Analysis of correlation between arterial travel time and detector data from simulation and field studies," Transportation Research Record, pp. 166-173, 1994.

[4] C. P. Young, "A relationship between vehicle detector occupancy and delay at signal-controlled junctions," Traffic Engineering and Control, vol. 29, pp. 131-134, 1988.

[5] C. Xie, R. L. Cheu, and D. H. Lee, "Calibration-free arterial link speed estimation model using loop data," Journal of Transportation Engineering, vol. 127, pp. 507-514, 2001.

[6] H. M. Zhang, "Link-journey-speed model for arterial traffic," Transportation Research Record, pp. 109-115, 1999.

[7] V. P. Sisiopiku and N. M. Rouphail, "Toward the use of detector output for arterial link travel time estimation: a literature review," Transportation Research Record, pp. 158-165, 1994. 